\title{
SPHERICAL DISTRIBUTIONS OF $N$ POINTS WITH MAXIMAL DIST ANCE SUMS ARE WELL SPACED
}

\author{
KENNETH B. STOLARSKY
}

ABSTRACT. It is shown that if $N$ points are placed on the unit sphere in Euclidean 3-space so that the sum of the distances which they determine is maximal, then the distance between any two points is at least $2 / 3 N$. Results for sums of $\lambda$ th powers of distances are also given.

The following problem is open: How should one place $N$ points $p_{1}$, $\cdots, p_{N}$ on the surface of the unit sphere $U$ in $E^{3}$ (i.e. on $x^{2}+y^{2}+z^{2}=1$ ) so that the sum of the distances which they determine is maximal? Many closely related problems have been studied; see for example [1]-[10]. Here we show that the points must be "well spaced" in the sense that no two can be closer than a distance of $2 / 3 N$. In fact, we will show a bit more. Let $\left|p_{i}-p_{j}\right|$ denote the Euclidean distance between $p_{i}$ and $p_{j}$.

Theorem. Let $0<\lambda<2$. If $p_{1}, \cdots, p_{N}$ are placed on $x^{2}+y^{2}+z^{2}=1$ so that

$$
\sum_{i<j}\left|p_{i}-p_{j}\right|^{\lambda}
$$

is maximal, then for $i \neq j$ we have

$$
\left|p_{i}-p_{j}\right| \geq\left[4 \lambda / 2^{\lambda}(2+\lambda)\right]^{1 /(2-\lambda)} N^{-1 /(2-\lambda)} \text {. }
$$

We can improve the constant here, but it is more important to improve the exponent of $N$. Since $\min \left|p_{i}-p_{j}\right| \ll N^{-1 / 2}$ can be shown easily by the pigeonhole principle for any distribution of $N$ points on this sphere, the exponent is almost best possible for $\lambda$ small. However, it is possible (and suggested by a result of Björck; see [3, P. 261, Remark 1]) that $\left|p_{i}-p_{j}\right|$ $\gg N^{-1 / 2}$ for $0<\lambda<2$.

It is interesting to observe that spherical distributions of points with maximal spherical distance sums are not necessarily well spaced [6], [8].

For the proof of the Theorem let $p_{1}, \cdots, p_{N}$ maximize the sum

Received by the editors November 20, 1973 and, in revised form, February 12, 1974 .

AMS (MOS) subject classifications (1970). Primary 52A25, 52A40. 


$$
\sum_{i<j} G\left(\left|p_{i}-p_{j}\right|\right)
$$

where $G$ is a continuous function defined on $[0,2]$ which has a fourth derivative everywhere in $(0,2)$. We will choose $G$ later. Rotate $U$ so that $p_{1}$ coincides with the north pole $(0,0,1)$. I.et

$$
f(p)=\sum_{i=2}^{N} G\left(\left|p-p_{i}\right|\right)
$$

and let $C$ be a circle on $U$ centered at $p_{1}$. Clearly $f\left(p_{1}\right)$ must be at least as large as the average value of $f(p)$ on $C$. Choose spherical coordinates $(\theta, \phi)$ so that any point $p=(x, y, z) \in U$ is given by $(\sin \phi \sin \theta, \sin \phi \cos \theta$, $\cos \phi)$. Thus

$$
\frac{1}{2 \pi} \int_{0}^{2 \pi} f(p) d \theta \leq f(0,0,1)
$$

Next, let $g=g(x, y, z)$ be any function with a convergent power series expansion

$$
g(x, y, z)=\sum_{i, j, k} c_{i j k} x^{i} y^{j}(z-1)^{k}
$$

about $(0,0,1)$. For $(x, y, z) \in C \subseteq U$, one has for $\phi$ small,

$$
\begin{aligned}
\Delta & \equiv \frac{1}{2 \pi} \int_{0}^{2 \pi} g(x, y, z) d \theta-g(0,0,1) \\
& =\sum_{i, j, k} c_{i j k}(\sin \phi)^{i+j}(\cos \phi-1)^{k} \frac{1}{2 \pi} \int_{0}^{2 \pi} \sin ^{i} \theta \cos ^{j} \theta d \theta .
\end{aligned}
$$

Now the integral on the right of (7) is zero unless $i$ and $j$ are both even. Hence when $\phi$ is small,

$$
\Delta=1 /\left.4 \phi^{2}\left(\frac{\partial^{2} g}{\partial x^{2}}+\frac{\partial^{2} g}{\partial y^{2}}-2 \frac{\partial g}{\partial z}\right)\right|_{p_{1}}+O\left(\phi^{4}\right) .
$$

In fact (8) is clearly valid under the weaker assumption that all partial derivatives of $g$ of order at most four exist.

Let $q=\left(x_{0}, y_{0}, z_{0}\right) \in U$ be fixed. We now set $g(p)=G(u)$, where $u=|p-q|$. Then

and

$$
\frac{\partial g}{\partial x}=\frac{d G}{d u} \frac{\left(x-x_{0}\right)}{|p-q|}
$$

$$
\frac{\partial^{2} g}{\partial x^{2}}=\frac{d^{2} G}{d u^{2}} \frac{\left(x-x_{0}\right)^{2}}{|p-q|^{2}}+\frac{d G}{d u} \frac{\left(y-y_{0}\right)^{2}+\left(z-z_{0}\right)^{2}}{|p-q|^{3}} .
$$

Upon evaluating partials at $p=p_{1}$, we find that the coefficient of $1 / 4 \phi^{2}$ in (8) is 


$$
\left(1-\frac{u^{2}}{4}\right) \frac{d^{2} G}{d u^{2}}+\left(\frac{1}{u}-\frac{3}{4} u\right) \frac{d G}{d u}
$$

where $u=\left|p_{1}-q\right|$. Thus

$$
\Delta=1 / 4 \phi^{2}\left\{\frac{1}{u} \frac{d}{d u}\left[u\left(1-\frac{u^{2}}{4}\right) \frac{d G}{d u}\right]\right\}+O\left(\phi^{4}\right)=a(u) \phi^{2}+O\left(\phi^{4}\right) .
$$

We now replace $q$ by $p_{i}$, where $2 \leq i \leq N$, and set $u_{i}=\left|p_{1}-p_{i}\right|$ so (9) becomes

$$
\Delta_{i}=1 / 4 a\left(u_{i}\right) \phi^{2}+O\left(\phi^{4}\right) .
$$

Assume now that the sum (4) is maximal. Let $s$ be the number of points $p_{i}$ which coincide with $p_{1}$, and let every point on $C$ make the small angle $\phi$ with $(0,0,1)$. We apply (5), (7), (9), and (10) with $g$ replaced by $f$ (recall (4)), and obtain

$$
\Delta=s \cdot 2 \pi \sin \phi+\phi^{2} \sum_{i=2}^{N} a\left(u_{i}\right)+O\left(\phi^{4}\right) \leq 0 .
$$

Here the dashed sum is taken over those $i$ for which $p_{i} \neq p_{1}$. By letting $\phi \rightarrow 0$ we see first that $s=0$, so the sum is over all $i \neq 1$. We then see that the sum is at most zero, i.e.

$$
\sum_{i=2}^{N} a\left(\left|p_{1}-p_{i}\right|\right) \leq 0 \text {. }
$$

If $G(u)=u^{\lambda}$, where $0<\lambda<2$, then

$$
a(u)=\lambda^{2} / 4 u^{2-\lambda}-\lambda(\lambda+2) u^{\lambda} / 16 \text {. }
$$

Thus for any $j \neq 1$ we have

$$
\begin{aligned}
\lambda^{2}\left|p_{1}-p_{j}\right|^{\lambda-2} & \leq \sum_{i=2}^{N} \lambda^{2}\left|p_{1}-p_{i}\right|^{\lambda-2} \\
& \leq 1 / 4 \lambda(2+\lambda) \sum_{i=2}^{N}\left|p_{1}-p_{i}\right|^{\lambda} \leq 1 / 4 \lambda(2+\lambda) 2^{\lambda} \cdot N .
\end{aligned}
$$

Since $p_{1}$ was chosen arbitrarily, this completes the proof of the Theorem.

One can now prove many theorems of this type by varying the choice of $G$. If we take

$$
G(u)=\int_{0}^{u} t h(t) \ln \left\{\frac{u}{t} \frac{\left(4-t^{2}\right)^{1 / 2}}{\left(4-u^{2}\right)^{1 / 2}}\right\} d t,
$$

then a purely formal calculation gives $a(u)=1 / 4 h(u)$. Thus if the integral 
(13) converges for $h(t)$, we have

$$
\sum_{i=2}^{N} h\left(\left|p_{1}-p_{i}\right|\right) \leq 0
$$

whenever the sum (3) is maximal. In the case $G(u)=u$, we have $h(t)=t^{-1}-$ $3 / 4$. We shall now choose $h(t)$ so that (14) is as strong as possible.

It is not hard to show there is a function $w(t)$ continuous on the closed interval $[0,2]$ such that (13) converges when $\epsilon>0$ and

$$
h(t)=t^{-2}(\ln (1 / t))^{-2}(\ln \ln (1 / t))^{-1-\epsilon}-w(t) .
$$

(In the case $G(u)=u$, the role of $w(t)$ was played by $3 / 4 t$.) It follows (from (14); we suppress the details) that if (3) is maximal, then for $i \neq j$ we have

$$
\left|p_{i}-p_{j}\right| \gg N^{-1 / 2}(\ln N)^{-1}(\ln \ln N)^{-1 / 2-10 \epsilon} \text {. }
$$

Here $G(u)$ behaves like $\epsilon^{-1}(\ln \ln (1 / u))^{\epsilon}$ as $u$ approaches 0 from the right. However, we know of no continuous $G$ for which (3) implies $\left|p_{i}-p_{j}\right| \gg N^{-1 / 2}$.

\section{REFERENCES}

1. R. Alexander, On the sum of distances between $n$ points on a sphere, Acta Math. Acad. Sci. Hungar. 23 (1972), 443-448. MR 47 \#957.

2. Ralph Alexander and K. B. Stolarsky, Extremal problems of distance geometry related to energy integrals, Trans. Amer. Math. Soc. 193 (1973), 1-31.

3. G. Björck, Distributions of positive mass, which maximize a certain generalized energy integral, Ark. Mat. 3 (1955), 255-269. MR 17, 1198.

4, L. Fejes Tóth, On the sum of distances determined by a pointset, Acta. Math. Acad. Sci. Hungar. 7 (1956), 397-401. MR 21 \#5937.

5. E. Hille, Some geometric extremal problems, J. Austral. Math. Soc. 6 (1966), 122-128. MR 33 \#6507.

6. F. Nielsen, Om summen af afstandene mellem n punkter pa en kugleflade, Nordisk Mat. Tidskr. 13 (1965), 45-50.

7. G. Pólya and G. Szegö, Über den transfiniten Durchmesser (Kapazitäts. konstante) von ebenen und räumlichen Punktmengen, J. Reine Angew. Math. 165 (1931), 4-49.

8. G. Sperling, Lösung einen elementargeometrischen Frage von Fejes Tóth, Arch. Math. 11 (1960), 69-71. MR 22 \#2935.

9. K. B. Stolarsky, Sums of distances between points on a sphere, Proc. Amer. Math. Soc. 35 (1972), 547-549. MR 46 \#2555.

10. - Sums of distances between points on a sphere.II, Proc. Amer. Math. Soc. 41 (1973), 575-582. 61801

DEPARTMENT OF MATHEMATICS, UNIVERSITY OF ILLINOIS, URBANA, ILLINOIS

Current address: Department of Mathematics, University of Colorado, Boulder, Colorado 80302 